Supporting Information

\title{
Stimuli-responsive extraction and ambidextrous redispersion of zwitterionic amphiphile-capped silver nanoparticles
}

\author{
Clara Morita-Imura*, Katsuya Zama, Yoshiro Imura, Takeshi Kawai, and Hitoshi Shindo* \\ Department of Applied Chemistry, Chuo University, Kasuga, Bunkyo-ku, Tokyo 112-8551 (Japan) \\ *cimura@kc.chuo-u.ac.jp, shindo@kc.chuo-u.ac.jp
}

\section{Differential Scanning Calorimetry (DSC) measurements}

To obtain the amount of C16CA ligand on the redispersed AgNPs, DSC measurements were carried out using a differential scanning calorimeter DSC8000 (Perkin Elmer).

$30 \mathrm{~mL}$ of $100 \mathrm{mM}$ aq. C16CA was added to $30 \mathrm{~mL}$ of the as prepared AgNPs dispersion, and the recovery-redispersion procedure was done as same as main article. Prior to all the measurements, free C16CA molecules were removed by centrifuging procedure (15000 rpm, $20 \mathrm{~min})$. Dried C16CA-AgNPs were sealed into $\mathrm{Al}$ pans and heated from 30 to $150{ }^{\circ} \mathrm{C}$ at the rate of $20{ }^{\circ} \mathrm{C} / \mathrm{min}$ under $\mathrm{N}_{2}$ gas. An empty Al pan was used as a reference.

The endothermic peaks at $\sim 109{ }^{\circ} \mathrm{C}(\Delta H=9.1 \mathrm{~kJ} / \mathrm{mol})$ and at $\sim 44{ }^{\circ} \mathrm{C}(\Delta H=11.1 \mathrm{~kJ} / \mathrm{mol})$ were originated from the phase transition of anionic- and zwitterionic C16CA, respectively (Figs. S1a, c). The amount of C16CA ligand on AgNPs was calculated from these enthalpy, as $3.8 \mu \mathrm{mol}$ in water-rediepersed AgNPs (Fig. S1b), $1.7 \mu \mathrm{mol}$ in chloroform-redispersed AgNPs (Fig. S1d), and $0.02 \mu \mathrm{mol}$ in methanol-precipitated AgNPs (Fig. S1e), respectvely. If all of the AgNPs were $11 \mathrm{~nm}$-diametered sphere, the adsorbed mole of C16CA ligand on AgNPs was estimated as $\sim 23 \mu \mathrm{mol} / \mathrm{m}^{2}$ in in water, $\sim 10 \mu \mathrm{mol} / \mathrm{m}^{2}$ in chloroform, and $\sim 1.2 \mu \mathrm{mol} / \mathrm{m}^{2}$ in methanol, respectvely. 


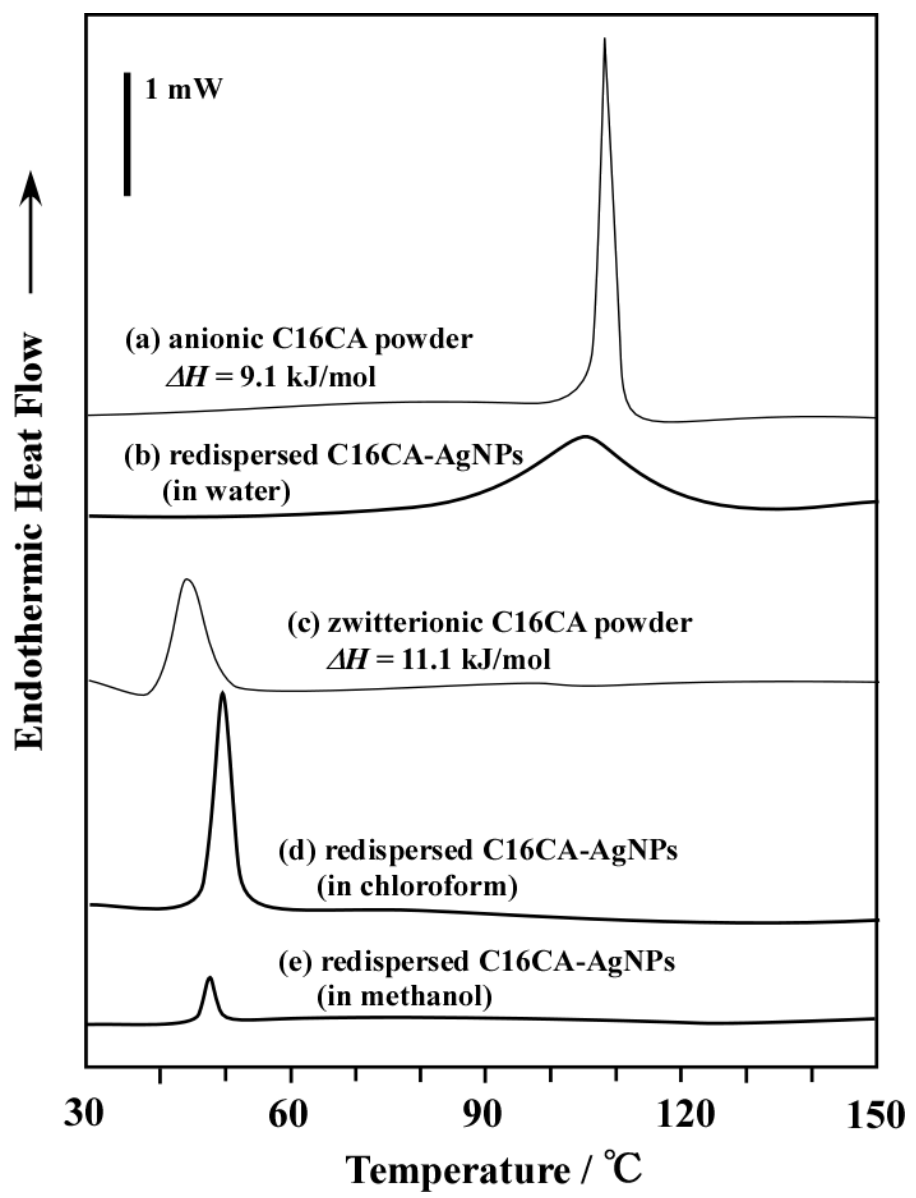

Figure S1. DSC thermograms of redispersed AgNPs in water (pH10), chloroform, and methanol.

Table S1. Summary of transition temperature of C16CA.

\begin{tabular}{|c|c|c|c|}
\hline & $\begin{array}{c}\text { Transition } \\
\text { temperature } /{ }^{\circ} \mathrm{C}\end{array}$ & $\begin{array}{c}\Delta \boldsymbol{H} \\
/ \mathrm{kJ} \cdot \mathrm{mol}^{-1}\end{array}$ & $\begin{array}{l}\text { Area } \\
\text { / } \mathbf{m J}\end{array}$ \\
\hline Anionic C16CA powder & 109 & 9.1 & 24.9 \\
\hline $\begin{array}{l}\text { Redispersed C16CA-AgNPs } \\
\text { (in water) }\end{array}$ & 105 & & 34.3 \\
\hline Zwitterinic C16CA powder & 44 & 11.1 & 30.5 \\
\hline $\begin{array}{l}\text { Redispersed C16CA-AgNPs } \\
\text { (in chloroform) }\end{array}$ & 49 & & 18.5 \\
\hline $\begin{array}{l}\text { Redispersed C16CA-AgNPs } \\
\text { (in methanol) }\end{array}$ & 47 & & 2.2 \\
\hline
\end{tabular}

